Revue d'histoire de l'Amérique française

RAS REVUE D.HISTOIRE DE L'AMÉRIQUE FRANÇAISE

\title{
Le rôle du blé à Montréal sous le régime seigneurial
}

\section{Corinne Beutler}

Volume 36, numéro 2, septembre 1982

URI : https://id.erudit.org/iderudit/304049ar

DOI : https://doi.org/10.7202/304049ar

Aller au sommaire du numéro

Éditeur(s)

Institut d'histoire de l'Amérique française

ISSN

0035-2357 (imprimé)

1492-1383 (numérique)

Découvrir la revue

Citer cet article

Beutler, C. (1982). Le rôle du blé à Montréal sous le régime seigneurial. Revue d'histoire de l'Amérique française, 36(2), 241-262.

https://doi.org/10.7202/304049ar d'utilisation que vous pouvez consulter en ligne.

https://apropos.erudit.org/fr/usagers/politique-dutilisation/ 


\title{
LE RÔLE DU BLÉ À MONTRÉAL SOUS LE RÉGIME SEIGNEURIAL*
}

\author{
CORINNE BEUTLER \\ Centre national de la Recherche scientifique \\ Centre de Recherches historiques de Paris
}

L'analyse de la situation du blé à Montréal sous le régime seigneurial constitue à plus d'un titre un point de référence indispensable à une réflexion sur les modèles d'approvisionnement durant cette période. Consommateurs de pain avant d'être producteurs de blé, les colons de la Nouvelle-France ont commencé par connaître, à l'égard de la métropole, une position de dépendance alimentaire qui s'est muée en une dépendance économique lorsqu'ils ont commercialisé par la suite le surplus de leur production céréalière. Des pays pauvres aujourd'hui, même en dehors d'un régime colonial révolu, connaissent parfois simultanément cette double dépendance imposée par la loi des plus forts sur le marché international. Nous nous proposons d'examiner pour commencer les fonctions du blé dans une colonie qui devient productrice puis exportatrice de céréales, et de suivre les filières de l'approvisionnement de Montréal jusque vers 1840 , en utilisant en particulier les comptes des moulins banaux du Séminaire de Saint-Sulpice de Montréal.

\section{Les rôles du blé}

Avant d'être cultivé en quantité suffisante pour répondre à la consommation locale, le blé, ou peut-être plus précisément la farine, était importé de France, comme l'illustre la présence d'un premier boulanger à Québec, signalée quelque six ans avant les débuts de l'agriculture locale ${ }^{1}$. Onze ans après le règlement qui imposait la banalité à tous les moulins à vent et à eau, l'arrêt de 1686 confirmait l'essor des défrichements et l'extension prise par la culture du froment. Cet arrêt faisait obligation aux seigneurs, détenteurs d'un fief dans l'étendue du pays, d'y construire un moulin banal en l'espace d'une année, faute de quoi le droit de banalité appartiendrait aux particuliers qui construiraient des moulins à farine ${ }^{2}$. L'accroissement de la production céréalière, en

* Le sujet de cette étude a été inspiré par le thème de recherche sur le stockage et la police des grains, présenté à la IIIe réunion internationale sur les Techniques de conservation des grains à long terme, Levroux, 24-29 novembre 1980.

Il est question, en 1620, d'une maison de la basse-ville où devaient loger le serrurier et le boulanger; voir J.-N. Fauteux, Essai sur l'industrie au Canada sous le régime français (Québec, 1927), II: 366.

${ }_{2}$ Edits, ordonnances royaux... concernant le Canada; arrêts et règlements du Conseil supérieur; complément des ordonnances et jugements des gouverneurs et intendants du Canada... (Québec, 1854-1856), I: 255-56. 
l'absence de débouchés assurés, engagea les curés et les missionnaires à se plaindre au roi de l'insuffisance de leurs ressources fondées sur les dîmes en nature, car, écrivaient-ils au début du XVIIIe siècle, «toutes les denrées qui croissent dans le pays se donnent à un prix fort médiocre faute de consommation» ${ }^{3}$.

Le rôle de monnaie d'échange joué par le blé dans des transactions intérieures jusqu'à la fin du XVIIIe siècle, au moins, est certes le reflet d'un surplus disponible par rapport aux besoins de la population; il signifie également que, dans une colonie où le numéraire était rare, le blé qu'ils produisaient représentait pour les paysans une des seules monnaies d'échange dont ils disposaient. En 1669, par exemple, dans cette société encore essentiellement rurale, un arrêt faisait obligation aux créanciers d'accepter en paiement le blé de leurs débiteurs pendant trois mois à dater de la publication de l'arrêt ${ }^{4}$. Quelque dix ans plus tard, une ordonnance, qui enjoignait aux habitants de se pourvoir d'un fusil pour faire face aux attaques surprises des Iroquois, invitait le «sieur Le Bert marchand de Montréal... de leur en Vendre et prendre en payement du bled bon loyal et marchand a raison de cinquante sols le Minot au moins, Et en chairs de cochon salée au prix ordinaire», pour ceux qui ne pourraient payer autrement ${ }^{5}$. Les études qu'a faites Louis Michel de deux marchands ruraux de Varennes ${ }^{6}$ font clairement apparaître la place qu'occupaient encore à la fin du XVIIIe siècle les paiements en blé dans des transactions courantes entre les paysans et des marchands locaux, qui, par ailleurs, n'étaient nullement des commerçants en grain. Nous ignorons malheureusement à quel prix et à la suite de quels marchandages se faisaient ces échanges.

\section{Le commerce international du blé}

La permanence d'un régime de troc signifiait-elle, pour autant, que la région vivait en marge du grand commerce céréalier? Certainement pas. L'entrée de la Nouvelle-France dans le commerce des farines, au début du XVIIIe siècle, n'avait pu que stimuler l'agriculture sur les rives fertiles du Saint-Laurent. On exporta les surplus d'abord vers les autres colonies non productrices de blé, comme l'Acadie et Terre-Neuve, puis pour ravitailler la popula-

3 Ibidem, I: 305-1.1.

4 Ibidem, II: 4.

5 Ordonnance du 24 octobre 1682, Québec (Prov.), Archives, Ordonnances, commissions, etc. etc. des gouverneurs et intendants de la Nouvelle-France, 1639-1706 (éd. P.-G. Roy, Beauceville, 1924), II: 12.

6 L. Michel, «Un marchand rural en Nouvelle-France, François-Augustin Bailly de Messein, 1709-1771», RHAF, 33, 2 (septembre 1979): 215-262, et "Le livre de compte (1784-1792) de Gaspard Massue, marchand à Varennes", Histoire sociale, Social History, XIII, 26 (novembre 1980): 369-398. 
tion croissante de Louisbourg et son importante garnison, et, de là, pousser jusqu'aux Antilles. Après la Conquête, et tant que l'Angleterre n'y eut pas porté un coup d'arrêt pour des raisons politiques, le Bas-Canada exporta ses surplus céréaliers en Espagne et au Portugal. La politique européenne, en forçant la métropole à s'approvisionner au Canada, contribua durant une vingtaine d'années à la fortune des marchands de Montréal. Ceux-ci, malgré le caractère protectionniste des Corn Laws, dont la mise en application n'intervint d'ailleurs que vers 1820 , se virent favorisés par les circonstances et par leur position-clé sur l'axe du Saint-Laurent, entre le nord des États-Unis et le port de Québec ${ }^{7}$. Il n'est toutefois pas possible de déterminer la part prise par les blés de la région montréalaise dans l'ensemble des exportations bas-canadiennes signalées par F. Ouellet ${ }^{8}$. Enfin, la fourniture de vivres aux armées, durant la Révolution américaine et la guerre de 1812, contribua à créer sur place des débouchés, que le bond en avant de l'immigration et le développement du commerce du bois allaient renouveler et multiplier ${ }^{9}$.

Dès 1734 , faisant suite au règlement rendu par le roi à propos du commerce des farines du Canada avec l'Isle royale et les îles françaises de l'Amérique, l'ordonnance qui attribuait des cribles cylindriques aux principaux moulins du gouvernement de Québec était étendue à ceux du gouvernement de Montréal. On y précisait que

les bleds sont encore bien plus abondants, et d'une meilleure qualité que dans les autres endroits de la colonie. Et afin que les négociants de la dite ville de Montréal puissent être en état comme ceux de Québec, d'entreprendre le commerce des farines par mer, sa Majesté a fait faire l'année dernière, un nouvel envoi de cribles cylindriques. ${ }^{10}$

Toutefois la situation géographique de Montréal, longtemps défavorisée par rapport à celle de Québec qui disposait seule d'un port sur l'océan, explique sa position de retrait vis-à-vis du grand commerce. «Les grosses transactions, écrit J. Mathieu, étaient finalement dans les ports terminaux; les autres relevaient d'une organisation interne, certes indispensable à l'armateur, mais dans une structure économique généralement extérieure au grand commerce.» ${ }^{11}$ Ce n'est qu'en 1832, en effet, après d'importants travaux

7 J. Boucher, «Les aspects économiques de la tenure seigneuriale au Canada (1760-1854)», in Recherches d'histoire économique (Paris, 1964), 190.

8 F. Ouellet, Le Bas-Canada, 1791-1840 (Ottawa, 1976), 495.

9 G. Paquet et J.-P. Wallot, «Crise agricole et tensions socio-ethniques dans le Bas-Canada, 1802-1812: éléments pour une réinterprétation"), RHAF, 26, 2 (septembre 1972): 204 et 215.

10 Edits, ordonnances royaux..., op. cit., II: 363.

1 J. Mathieu, Commerce entre la Nouvelle-France et les Antilles au XVIIIe siècle (Montréal, 1981), 121 . 
d'aménagement, que le port de Montréal pourra prétendre à devenir un port d'entrée ${ }^{12}$. C'est pourquoi, tout en étant au coeur d'une région plus propice à la céréaliculture que celle de Québec, Montréal a pu se trouver dans la position périphérique décrite par J. Mathieu, tant qu'elle ne présida pas directement à l'exportation de ses blés, ou qu'elle ne devint le centre de transit et plus tard de transformation des blés de l'Ouest canadien et du nord des ÉtatsUnis.

La correspondance entre des marchands de Québec et leurs fournisseurs en blé de Montréal, en particulier celle des administrateurs de l'île Jésus avec le Séminaire de Québec au début du XIXe siècle ${ }^{13}$, atteste l'ancienneté et l'importance de ce courant commercial entre les deux villes. Mais il n'est pas encore possible, aujourd'hui, de suivre avec précision ni d'évaluer le mouvement de ces transactions, à cause de la dispersion des sources dans les correspondances de l'époque et des difficultés au niveau de l'interprétation statistique. Les données dont nous disposons sont celles des exportations au port de Québec, encore une fois, et comme on ne précisait pas la provenance de ces blés, nous ignorons la proportion de ceux de Montréal. L'activité du trafic fluvial entre les deux villes, signalée par une ordonnance de 1750 «rendue au sujet des maîtres de barques qui veulent s'approprier le bénéfice du bled qu'ils chargent», ne nous renseigne pas sur les quantités que des "particuliers font venir des côtes de Montréal à Québec pour leur commerce ${ }^{14}$. Ce prétendu bénéfice, que l'intendant contestait aux maîtres de barques qu'il accusait de vol manifeste, provenait de l'augmentation temporaire du volume du blé de plus de $6 \%$, provoquée par l'humidité durant le transport. Jusqu'à la mise en service des bateaux à vapeur, dont le premier date de 1809 , le transport en effet était très lent. Pour remonter le Saint-Laurent de Québec à Montréal, par exemple, un bateau pouvait mettre un mois en cas de défaillance du vent. Ce n'est qu'à partir de l'Acte de 1841 pour pourvoir à l'amélioration et à l'agrandissement du Havre de Montréal, qu'il fut prévu un enregistrement du trafic fluvial, en vue d'appliquer des droits de quayage.

\section{Le commerce local du blé}

L'aménagement de la première place du marché à Montréal date du début du XVIIIe siècle, c'est-à-dire plus de cinquante ans

12 P. Brouillard, «Les archives du port de Montréal», in Rapport et Travaux 197375, du Groupe de Recherche sur la société montréalaise au 19e siècle (GRSM), (Dpt d'Histoire de l'UQAM, 1975), VI: 1.

13 S. Depatie, L'administration de la seigneurie de l'île Jésus au XVIIIe siècle (mémoire de maîtrise, Dpt d'Histoire de l'Université de Montréal, 1979).

1414 août 1750 , Edits, ordonnances royaux..., op. cit., II: 403 . 
après la fondation de la ville. Contrairement à Québec, Montréal n'a pas construit de halle aux grains; elle n'a, par ailleurs, envisagé à aucun moment l'organisation d'un réseau de greniers publics qui auraient assuré l'approvisionnement du marché en temps normal ou en cas de crise, comme cela se faisait en Europe occidentale ${ }^{15}$. Par contre, en période de guerre, on entreposait dans les magasins du roi, à Québec et à Montréal, les blés et les farines du roi confiés aux munitionnaires. Même si elles sont destinées aux troupes, «ces farines venues de France, écrit J.-E. Labignette, serviront de volant sur le marché de la farine lors des fluctuations saisonnières des cours.» 16

Les transactions de blé et de farine sur la place du marché de Montréal ne nous sont connues que par les dispositions, inspirées du règlement de Québec de 1676, qui concernent l'utilisation des mesures à grains de toutes sortes (le minot égal à 39 litres, et le demi-minot), et par celles, très détaillées, du règlement de 1810 , établi pour l'ouverture du deuxième marché de Montréal. Il y est précisé, à l'article 50, que le clerc du marché doit assister aux transactions, mesurer lui-même le grain avec les vieilles mesures françaises aux frais du vendeur, lorsqu'il y est requis par l'acheteur, et s'assurer de la contenance des poches pour la farine, fixée en mesures anglaises (120 pounds pour la farine et 112 pour la fleur, non compris le poids de la poche) ${ }^{17}$. Si l'on songe à la réglementation complexe, parfois contradictoire, qui devait assurer un achalandage régulier des marchés parisiens tout en excluant le stockage prévisionnel en ville, ainsi qu'à l'initiative municipale, caractéristique au contraire des villes du Saint-Empire dans leur ensemble, on est frappé par la liberté qui présidait à l'approvisionnement de Montréal. Cette liberté du marché intérieur n'était cependant pas imputable à des circonstances plus favorables qu'ailleurs, puisque, pendant longtemps la colonie n'a pu se suffire à elle-même. Un mémoire, adressé au roi en 1673 , caressait le projet encore très optimiste «que le Canada pourra se décharger chaque année de trente mil minots les quatre faisant le setier de Paris, si par l'Inclémence du ciel les récoltes ne sont pas disgraciées» ${ }^{18}$.

15 S. L. Kaplan, «Lean Years, Fat Years: the 'community' granary system and the Search for Abundance in Eighteenth-Century Paris», French Historical Studies, X, 2 (1977): 197-230. Et C. Beutler, «De l'approvisionnement en grains de quelques villes européennes au Moyen âge et à l'époque moderne», in Les techniques de conservation des grains à long terme, I (Paris, CNRS, 1979): 95-104. 1964): 490-503.

J.-E. Labignette, «La farine dans la Nouvelle-France», RHAF, 17, 4 (mars

17 J. Brown, Règles et règlements de Police pour la ville et les faubourgs de Montréal. Approuvé et confirmé par les juges de la Cour du Banc du Roi, dans le terme de février 1810 (éd. bilingue, Montréal, 1810).

18 J.-E. Labignette, op. cit.: 493. 
Malgré l'extension des défrichements dans le bassin du SaintLaurent, il fallait faire appel à l'aide de la métropole lors des accidents climatiques, parfois catastrophiques. Le rythme des naissances et les vagues successives d'immigration, qui, théoriquement, devaient multiplier la main-d'oeuvre et les débouchés, ne furent pas sans poser des problèmes d'approvisionnement à une région où l'agriculture traditionnelle était particulièrement sensible aux variations climatiques. Cependant, dans le cas qui nous occupe, il faut observer avec Louise Dechêne que «tout au long du XVIIIe siècle, après comme avant la Conquête, Montréal se développe à un rythme très lent» ${ }^{19}$. Développement suffisant toutefois, au début du XVIIIe siècle surtout, pour justifier l'ouverture d'un premier marché «tant pour procurer à ses habitans le plus d'abondance que faire se pourra, que pour donner à ceux de la campagne la facilité qu'ils peuvent désirer pour vendre leurs denrées» ${ }^{20}$.

Le premier règlement de police faisait obligation aux paysans d'exposer leurs produits au marché avant d'aller les proposer dans les maisons des particuliers; il accordait à ces derniers une heure d'avance sur les professionnels pour faire leurs emplettes au marché, ainsi que la liberté de s'approvisionner directement à la campagne s'ils le désiraient. Après la Conquête, les nouvelles dispositions ajoutèrent aux précédentes l'interdiction faite aux paysans de vendre sur les chemins ou à l'abordage de leurs canots au port de Montréal; en outre le marché, de bi-hebdomadaire qu'il avait été initialement, se tint désormais trois fois la semaine. Alors que la boucherie, autant peut-être pour surveiller le commerce d'une production locale insuffisante que pour des raisons d'hygiène publique, faisait l'objet d'une réglementation détaillée, et que les bouchers devaient louer leurs étaux à l'année pour vendre au marché, nous ne disposons d'aucune indication concernant l'emplacement et l'exposition des blés et des farines au marché. Nous ignorons si on y vendait du pain comme dans les marchés spécialisés de Paris, par exemple. Le clerc des marchés, en tout cas, ne louait pas de banc ni de place à la journée pour la vente des céréales, comme il le faisait pour d'autres produits. Il est vrai qu'une iconographie plus récente montre encore, à la fin du XIXe siècle, des paysannes qui se tiennent sur une place de marché en France tout simplement debout derrière leur sac de blé posé par terre ${ }^{21}$. Il semble que les

19 L. Dechêne, «La croissance de Montréal au XVIIIe siècle», RHAF, 27, 2 (septembre 1973): 165 et passim. Cf. son estimation du nombre d'habitants de la ville et des faubourgs de Montréal, d'après les recensements de la population canadienne et l'analyse des aspects et des implications de cette croissance.

20 Ordonnance du 22 juin 1706, pour l'établissement d'un marché sur la place d'Armes, Edits, ordonnances royaux..., op. cit. II: 261 .

${ }_{21}$ Camille Pissaro, «Étude pour le Marché aux blés à Pontoise», D.34, in Pissaro, monde rural, art, politique (Centre culturel du Marais, Paris, 1981), 37. 
places de marché à Montréal, contrairement à ce qui se passait en Europe en général, n'ont pas constitué le lieu privilégié du commerce intérieur des grains.

En 1667, les modifications apportées par le Conseil Supérieur à l'ordonnance ou code civil précisaient que:

en toutes nos villes et bourgs où il y aura marché, les marchands, faisant trafic de bleds et autres espèces de gros fuits, ou les mesureurs, feront rapport par chacune semaine de la valeur et estimation commune des fruits, sans prendre aucun salaire; à quoi faire ils pourront être contraints par amendes ou autres peines qui seront arbitrées par les juges.

Malgré cette ordonnance, nous ne disposons pas pour Montréal d'une mercuriale des prix, comme à Cologne depuis le XIVe siècle, ou à Paris depuis le XVIe ${ }^{22}$. La même indication rapportée par R. Shore Milnes ${ }^{23}$, suivant laquelle un arrêt en date du 20 décembre 1677 ordonnait que des «mercuriales seront tenues pour régler les matières de police» ne semble pas avoir eu d'effet. Quant à l'obligation faite aux boulangers de vendre leur pain aux prix et aux poids fixés deux fois par année, d'après le prix du minot de grain, elle aurait pu fournir, à partir de 1676, les séries de références indispensables pour connaître le prix du grain et les activités de marché, si les données nous en étaient parvenues au complet. Le règlement de police du 11 mai 1676 pour la ville de Québec prévoit, en effet, à l'article 42 , que «le 15 avril et le 15 novembre de chaque année le lieutenant-général tiendra une assemblée générale des principaux habitants de la ville. On y arrêtera le prix du pain»» ${ }^{24}$.

L'année suivante, sur les plaintes de plusieurs habitants de la ville de Québec, vraisemblablement contre les prix excessifs pratiqués par les boulangers, le Conseil Supérieur de Québec ordonnait que le «lieutenant-général, sans tirer à conséquence pour l'avenir, convoquera la dite assemblée d'habitans, fera faire l'essai du pain des boulangers et y mettra le prix» ${ }^{25}$. Le compte rendu détaillé d'une séance du Conseil Supérieur de Québec, au cours de laquelle on fixa le prix du pain en fonction du prix courant du minot de blé et de la quantité de pain blanc et bis-blanc qu'il produisit, figure dans

22 Procès-verbal du 7 novembre 1678, Edits, ordonnances royaux..., op. cit., I: 200, art. VI du titre 30. Pour la mercuriale de Cologne, cf. D. Ebeling et F. Irsigler, Getreideumsatz, Getreide - und Brotpreise in Köln (1368-1797), et Brotgewichte und Brotpreise: Wochen -, Monats - und Jahrestabelle, Graphiken (Böhlau-Verlag, Köln-Wien, 1976 et 1977), I et II. Pour la mercuriale de Paris, cf. M. Baulant et J. Meuvret, Prix des céréales extraits de la mercuriale de Paris (1520-1698) (Paris, 1960-62), vol. 1 et 2.

23 R. Shore Milnes, Ordonnances des Intendants et Arrêts portant Règlements du Conseil supérieur de Québec (Québec, 1806), II, Table des principaux arrêts, etc. dont l'impression a été omise.

${ }_{24}^{24}$ Edits, ordonnances royaux..., op. cit., II: 84 .

25 Ibidem. 
les attendus de l'arrêt du Conseil du 2 décembre 1715, dont voici des extraits:

cette épreuve a été faite sur du bled de mouturage qui étoit de la moindre qualité, et que néanmoins il a produit quatorze livres, treize onces et demie de pain blanc et quarante-une livres et demie de pain bis-blanc;... qu'ils (les boulangers) peuvent faire quatre pains blancs de fine fleur qui pèseront en tout quatorze livres, et sept pains bis-blancs qui pèseront en tout quarante-deux livres, moyennant quoi ils retireront onze livres du minot de bled (sur la base du prix courant du minot de blé qui est alors de huit livres) et auront encore de reste suivant l'épreuve ci-dessus cinq onces et demie de pain par minot, et beaucoup plus quand le bled sera meilleur, et outre cela tout le son. ${ }^{26}$

En conséquence de quoi le Conseil ordonna que les boulangers vendraient «le pain blanc de fine fleur, du poids de trois livres et demie pour vingts sols, et le pain bis-blanc du poids de six livres aussi pour vingt sols» ${ }^{27}$.

L'attention portée par le Conseil aux intérêts des boulangers s'expliquait par le fait que ceux-ci s'étaient plaints à leur tour d'être trompés dans leurs achats de blé, parce que les mesures n'étaient ni marquées ni étalonnées, et que des meuniers n'avaient pas de balance dans leurs moulins. Le dernier article de cet arrêt stipulait, en outre, que des copies du tout seraient envoyées à Montréal et à Trois-Rivières. Ce dernier point, même s'il concernait strictement l'«épreuve du pain» faite à Québec seulement, soulève la question des assemblées d'habitants; s'en tenait-il aussi à Montréal? Malgré l'affirmation de Parent, selon laquelle la "convocation d'habitants» sous le Régime français se tenait à Québec comme à Montréal, afin de faire l'examen du pain et d'en déterminer le prix ${ }^{28}$, nous n'avons pas encore trouvé de compte rendu ni de traces de telles assemblées à Montréal.

D'après les informations recueillies, le poids du grand pain blanc serait passé de 3 livres en 1688 à 3 et 6 livres en 1706, à $31 \frac{1}{2}$ en 1715 , pour être fixé à 4 livres à partir de 1764 , et le bis-blanc, qui pesait 4,8 et 12 livres en 1688, aurait un poids uniforme de 6 livres à partir de 1715. Parce qu'ils étaient fixés en relation avec le prix courant du minot de blé sous le Régime français, les prix à la livre variaient en conséquence. Ce système était à l'opposé de celui pratiqué à Cologne, par exemple, où, du XVe au XVIIIe siècle, la

26 Ibidem: 169-170.

27 Ibidem.

28 H. Parent, Petite histoire administrative de Montréal, 1642-1942 (Exemplaire dactylographié déposé à la Salle Gagnon de la Bibliothèque municipale de Montréal, 1942), période 1663-1760. 
taxation du pain (Brottaxe), décidée par les autorités de la ville en vue d'assurer son approvisionnement, consistait à «fixer le poids du pain que l'on peut théoriquement se procurer pour 1 billon, en fonction du prix des céréales ${ }^{29}$. L'évolution des poids des pains, couramment vendus au public, pourrait peut-être éclairer celle des habitudes alimentaires, et donner une idée de la composition-type des familles urbaines; mais il faudrait pour cela disposer de plusieurs points de comparaison. En 1695, par exemple, la ration quotidienne de pain allouée au soldat stationné au Canada était de $11 / 2$ livre, et en 1706, d'après un mémoire de l'intendant, l'habitant aurait mangé 2 livres de pain par jour ${ }^{30}$. Pour le dernier quart du XVIIIe siècle, enfin, "on admet que la ration annuelle de pain d'un homme adulte correspond à douze minots de blé» ${ }^{31}$. Si L. Dechêne calcule qu'il faut 7,1 minots de blé par année pour fournir la ration la plus faible, est-il légitime de penser que la consommation de pain ait autant augmenté, alors qu'on s'attendrait au contraire à une diversification de l'alimentation?

Après la Conquête, une ordonnance du gouverneur de la colonie de Québec, du 3 septembre 1764, confiait à trois juges de paix, dans les districts de Québec et de Montréal, le soin d'établir «tous les premiers lundis de chaque mois» l'assise et le poids du pain, à proportion «du prix de la fine fleur de farine» ${ }^{32}$. Cette disposition fut reprise par l'ordonnance du 29 mars 1777, qui réglait «les poids du pain... ayant toujours égard aux prix du bled et de la farine» ${ }^{33}$, ainsi que par les Statuts provinciaux de 1815. En fait, sur les neuf mentions examinées jusqu'à présent pour Montréal, de 1764 à 1819, nous n'avons trouvé qu'en 1764 seulement l'indication du prix de la farine, sans celle du blé d'ailleurs ${ }^{34}$ :

quand le prix de la fine fleur de farine est à, ou au dessous de quatorze chélins, les cent douze livres Avoir du poids, le pain de six sols de la dite fleur pesera 4 livres Avoir du poids, idem du pain bis, 6 livres. Et dans cette proportion du plus ou moins, quand la fine fleur de farine est plus ou moins chere que quatorze chélins les cent douze livres.

Ce dernier poids était officiellement celui de la poche de fine fleur vendue au marché. Par la suite, cependant, le poids des pains ne

29 F. Irsigler, «La mercuriale de Cologne (1531-1797); structure du marché et conjoncture des prix céréaliers», Annales (Économies Sociétés Civilisations), 33, 1 (janvierfévrier 1978): 94.

30 L. Dechêne, Habitants et marchands de Montréal au XVIIe siècle (Paris Montréal, 1974), 342 note 61 , et 343.

31 L. Michel, «Le livre de compte de Gaspard Massue...», op. cit., 379, note 31.

32 Ordonnances, faites pour la province de Québec par le gouverneur et Conseil de la dite province depuis l'établissement du gouvernement civil, (Québec, 1767), I: 15.

33 Les Actes et ordonnances révisés du Bas-Canada (Montréal, 1845), 625.

34 Ibidem. 
semble plus avoir été mis en cause, et seuls les prix varièrent. D'après les informations officielles publiées par le Montreal Herald du 20 mai 1815, il n'y aurait même plus eu que deux sortes de pains aux poids fixés une fois pour toutes, «the White Loaf, No 1, of 4 Lbs... 11 d., the Brown Loaf, No 2, of 6 Lbs... 1 s.» ${ }^{35}$

$\mathrm{Si}$, d'après les rapports mensuels du clerc du marché à compter d'avril $1805^{36}$ nous disposons, pour la ville de Québec, des prix courants sur le marché, mais non des quantités vendues, rien de tel pour Montréal. Les tables des prix du blé à Montréal de 1790 à 1825 , et des prix du blé vendu en ville de 1790 à 1862 ne doivent pas faire illusion, car elles indiquent le «prix commun du bled vendu en ville» ${ }^{37}$. Ce prix n'était pas nécessairement le prix du marché et représentait de surcroît l'estimation en argent faite par les seigneurs de Montréal, au mois de novembre, pour le blé de rentes qui ne leur avait pas été livré en nature au cours de l'année écoulée. Or ces estimations ne correspondaient pas toujours aux prix de vente du blé de mouture, de rentes et de dîmes pratiqués par le Séminaire, et qu'on peut calculer mensuellement d'après les États du bled vendu dans les moulins de l'île et au grenier des seigneurs. Le Quebec Mercury du 9 juin 1806 publiait les réflexions d'un correspondant anglophone sur les marchés de Montréal, qui dénonçaient l'extrême confusion qui y régnait. Il paraît significatif que la première proposition de ce correspondant ait concerné les blés, pour lesquels il souhaitait la construction d'un édifice tout à fait conforme au modèle européen traditionnel que nous connaissons:

On the ground of the ancient college I would build a wheat stall; it should be a low and covered building, on pillars, where each inhabitant, or merchant, should deposit his grain or sample, of whatever species it might be, and pay so much a bushel on the quantity he sells, for defraying the expense of the building. ${ }^{38}$

Il semblerait donc bien que, pour la vente des blés au marché, on n'ait disposé alors d'aucune installation particulière, ce qui pourrait faire penser que le commerce intérieur du blé bénéficiait d'une large liberté.

Cette liberté pouvait-elle à l'occasion être préjudiciable au public? Dans un cahier manuscrit, qu'on peut dater de 1811 et qui

35 L. M. Wilson, This was Montreal in 1814, 1815, 1816 and 1817, compiled by... (Éd. privée The Château Ramezay, Montréal, 1960), 25.

36 G. Paquet et J.-P. Wallot, «Aperçu sur le commerce international et les prix domestiques dans le Bas-Canada (1793-1812)», RHAF, 21, 3 (décembre 1967): 458-468.

37 Archives du Séminaire de Saint-Sulpice à Montréal (ASSSM), tiroir 91, no 7 et no 45. Nous remercions M. B. Harel, p.s.s., archiviste du Séminaire de Saint-Sulpice, pour l'aide qu'il a apportée à notre travail aux archives du Séminaire.

${ }_{38}$ The Quebec Mercury, Monday, June 9, 1806, II: 23. 
est consacré à l'administration des moulins banaux du Séminaire de Saint-Sulpice à Montréal, seigneur de l'île de Montréal et de deux autres seigneuries voisines, l'économe, M. Molin, p.s.s., notait que «les habitans n'en apportent pas assez (de blé) au marché pour les boulangers» ${ }^{39}$. Dans quelle perspective faut-il replacer cette remarque? L'année 1811 s'appuie sur la récolte de 1810 , qui a été faible, de l'avis de M. Séguin, de sorte que «le surplus disponible en 1811 fut par suite très restreint ${ }^{40}$. J. Boucher signale également qu' «en 1811, à cause des mauvaises récoltes (précédentes), les exportations furent insignifiantes, tandis qu'en 1812, elles atteignirent un nouveau sommet» ${ }^{41}$. Qu'en était-il, par contre, de la demande intérieure, celle du marché urbain? «Jusqu'en 1807, écrit F. Ouellet, le marché local demeure peu important; ce n'est qu'à partir de 1808-09 qu'il acquiert des proportions nouvelles.» ${ }^{42}$ Sans entrer dans l'analyse des causes qui ont diversifié les débouchés pour les producteurs, on peut en effet conclure avec G. Paquet et J.-P. Wallot que la demande locale était devenue de plus en plus importante ${ }^{43}$. Il suffit, pour s'en convaincre, de se reporter aux chiffres avancés pour la population de Montréal au début du XIXe siècle ${ }^{44}$ :

\begin{tabular}{|c|c|c|}
\hline Année & $\begin{array}{l}\text { Montréal } \\
\text { ville et faubourgs }\end{array}$ & Taux annuels d'accroissement \\
\hline$\overline{1805}$ & 9020 habitants & $\begin{array}{l}1,6 \% \text { pour la période } 1784- \\
1805 \\
2,8 \% \text { d'après l'estimation de } \\
\text { L. Dechêne pour } 1784\end{array}$ \\
\hline 1815 & 15000 habitants & $\begin{array}{l}5,2 \% \text { pour la période } 1805- \\
1815 \text {. }\end{array}$ \\
\hline
\end{tabular}

Le fort accroissement de la demande urbaine ne suffit cependant pas à expliquer l'insuffisance du blé au marché, puisque la faiblesse de la récolte précédente n'aurait dû normalement affecter que les exportations. Il est vrai que l'inflation, dont le sommet pour la période $1792-1812^{45}$ se situait précisément entre novembre

39 ASSSM, tiroir 91, no 40, vol. 3, fol. 1vo 2, point 23.

40 M. Seguin, La 'nation canadienne' et l'agriculture (1760-1850), essai d'histoire économique (Trois-Rivières, 1970), 79.

41 J. Boucher, op. cit., 190.

42 F. Ouellet, Histoire économique et sociale du Québec, 1760-1850, structure et conjoncture (Montréal, 1971), I: 181.

43 G. Paquet et J.-P. Wallot, «Crise agricole...», op. cit.: 211.

44 J.-P. Bernard, P.-A. Linteau, J.-Cl. Robert, "Les tablettes statistiques de Jacques Viger (1825)", in Rapport et Travaux 1972-73, du GRSM (Dpt d'histoire de l'UQAM, 1973), V: 2 .

45 G. Paquet et J.-P. Wallot, "Crise agricole...», op. cit., 224, Tableau de l'indice mensuel désaisonnalisé des prix agricoles dans la région de Montréal. 
1810 et février 1811 , pouvait ralentir la circulation des grains, car les habitants avaient "tendance à retenir leur blé le plus longtemps possible, au printemps, afin de forcer les exportateurs à hausser leurs prix» ${ }^{46}$. L'essentiel de l'explication, à notre avis, se situe ailleurs: il tient au rôle joué par les moulins banaux du Séminaire de Saint-Sulpice dans la filière d'approvisionnement en blé de Montréal.

\section{Les moulins du Séminaire de Saint-Sulpice}

La filiale canadienne du Séminaire de Saint-Sulpice à Paris s'était installée à Ville-Marie en 1657; en 1663, le Séminaire de Paris devenait officiellement seigneur de l'île de Montréal, puis ultérieurement de deux autres seigneuries, celle du lac des DeuxMontagnes au nord-ouest et celle de Saint-Sulpice au nord-est. Après la Conquête, le Séminaire de Montréal se sépara de celui de Paris, mais, malgré la rupture de ces liens, il eut à lutter pour la reconnaissance de ses anciens droits seigneuriaux. G. Paquet et J.-P. Wallot relèvent les effets négatifs de la contestation légale des droits seigneuriaux des Sulpiciens sur l'île de Montréal à partir de 1820 , en particulier sur le produit de leurs moulins banaux ${ }^{47}$. Les droits seigneuriaux seront abolis à Montréal en 1840, et en 1854 dans tout le Bas-Canada, mais les dernières questions litigieuses, comme le rachat des rentes foncières, ne seront définitivement réglées qu'en $1940^{48}$.

Les «Règles générales pour la conduite de tous les moulins», manuscrit rédigé par l'économe du Séminaire, cité plus haut, révèlent entre autres la préoccupation des seigneurs de Montréal de défendre leur droit de banalité, en insistant sur les services réels que leurs moulins banaux rendaient au public. Ces services étaient en fait de deux sortes: à celui du mouturage, imposé aux censitaires producteurs de blé, mais laissé libre et au choix des nonproducteurs qui faisaient moudre du blé acheté, s'ajoutait celui d'approvisionner en blé des particuliers, surtout des boulangers de la ville et des marchands. Ces approvisionnements se faisaient à partir des stocks constitués dans les moulins par le règlement en

46 Ibidem, 203 note 53.

47 Idem, «Rentes foncières, dîmes et revenus paysans: le cas canadien» (communication présentée au colloque international des historiens économistes sur les dîmes, Paris, juillet 1977), 10.

48 «Ordonnance pour incorporer les Ecclésiastiques du Séminaire de Saint-Sulpice de Montréal, pour confirmer leur titre au fief et seigneurie de l'Isle de Montréal..., pour pourvoir à l'extinction graduelle des redevances et droits seigneuriaux... 1840-41», in Les Actes et ordonnances révisés..., op. cit., classe K, no 42, 658 et sq.; V. Morin, Seigneurs et censitaires, castes disparues, par V.M. vice-président du Syndicat National du Rachat des Rentes seigneuriales (Montréal, 1941); et J.-P. Wallot, Un Québec qui bougeait, trame socio-politique du Québec au tournant du XIXe siècle (Québec, 1973), 225-251. 
nature du droit de mouture. Voici ce qu'en disait l'économe, M. Molin:

Plus les moulins sont entretenus en bon état, plus les habitants et les boulangers y vont en foule... Il faut vendre autant qu'on peut ce bled des moulins aux habitants, et bien mieux encore aux boulangers, les uns et les autres font gagner les moutures parce qu'ils font moudre leur bled... (tandis que) les marchands l'enlèvent pour l'exporter; il vaut même mieux le vendre toujours aux boulangers. ${ }^{49}$

C'était effectivement ce qui se passait au moulin de Lachine «qui est le plus à portée des boulangers de la ville, soit parce que les chemins sont presque toujours beaux et qu'il ne manque jamais d'eau pendant l'été, soit parce qu'on fait la plus grande partie du chemin sur la glace, et qu'avec un peu de précaution il ne s'arrête jamais pendant l'hiver»' ${ }^{50}$. Il en allait de même aux deux moulins du Sault-au Récollet, ainsi qu'à celui du Gros-Sault sur la rivière des Prairies, où, poursuivait l'économe, «il est rare que les boulangers de la ville n'achètent pas tout le bled de mouture dans ce moulin ${ }^{51}$. Quant aux «meuniers près de la ville», M. Molin recommandait qu'ils eussent «beaucoup d'égard aux boulangers, soit pour la farine fine ou grosse, soit pour ne pas les laisser manquer, afin que les particuliers de la ville ne soient pas mécontents» ${ }^{52}$.

Fixé une fois pour toutes au quatorzième minot, le droit de mouture n'a pas varié durant tout le régime seigneurial. Mesurés en grain avant le mouturage, ces prélèvements étaient déposés par le meunier dans un réduit fermant à clé, le "gargouchet». Chaque mois, en principe, le Séminaire réglait ses comptes avec ses meuniers, ce qui revenait à partager les grains de mouture accumulés au gargouchet, sur la base des conditions qui figuraient dans les baux. Les premiers paiements en argent apparurent sporadiquement à partir de 1823, d'abord au moulin à vent de Sainte-Anne. Lorsque le mode d'afferme du moulin ne reposait pas sur une quantité mensuelle (ou trimestrielle) fixe de blé réclamée par le Séminaire, la part qui revenait au meunier pour son salaire variait entre le quart, le tiers ou la moitié des grains de mouture. Le Séminaire faisait porter dans son grenier en ville le blé nécessaire à sa consommation et laissait le reste de sa part à vendre sur place aux moulins, qui tenaient ainsi lieu de magasins de grains. Pour éviter des tentations à ses meuniers dont les moulins pouvaient être fort

49 ASSSM, tiroir 91, no 40, vol. 3: points 21 et 24 .

50 Ibidem, moulin de La Chine, fol. $4 \mathrm{r}^{\circ} 2$ : point 1 .

51 Ibidem, moulins du Sault au Récollet, fol. A $\mathrm{r}^{\circ}$ : point 5, et moulin du Gros Sault, fol. $4 \mathrm{r}^{\circ}$ 1: point 13.

52 Ibidem, «Règles générales...»: point 38. 
éloignés de la ville de Montréal, et pour surveiller de plus près la marche de ses affaires, le Séminaire remettait en général à ses clients des «billets», que ceux-ci présentaient aux meuniers en allant chercher la quantité de blé mentionnée. Ils en réglaient ensuite le montant à l'économe.

Entre 1767 (date des premiers comptes sommaires avec les meuniers) et 1840 , c'est-à-dire durant presque trois quarts de siècle, le Séminaire a eu jusqu'à 16 moulins, dont cinq à eau et quatre à vent sur l'île même de Montréal. Le dernier moulin construit sur l'île, le moulin à eau du Gros-Sault, commença à moudre fin décembre 1800. Pour les 110 ans qui précèdent, on dénombre une vingtaine de moulins qui ont tourné sur l'île à un moment ou à un autre; mais leur identification et leur localisation précise font problème $^{53}$.

Les comptes des moulins que nous possédons, et qui mentionnent le nom et parfois la profession ou l'état des acheteurs, ne commencent qu'en 1795. Nous disposons, par contre, depuis 1767, de comptes plus sommaires, qui indiquent les quantités de blé tenues à la disposition du Séminaire par ses meuniers. Enfin, grâce aux baux que le Séminaire a passés devant notaire, nous pouvons nous faire une idée de la quantité de grain qui revenait théoriquement au Séminaire, dans le cas d'afferme à un prix fixe en natu$\mathrm{re}^{54}$. Aux séries complètes reposant sur les blés de mouture qui revenaient au Séminaire, il faut ajouter celles des blés de rentes en provenance de ses trois seigneuries, et celles des blés de dîmes pour l'île de Montréal seulement. La part des blés de rentes et de dîmes qui lui était versée en nature variait, mais elle n'était pas forcément négligeable. Le blé de rentes hors de l'île était ordinairement entreposé sur place dans un moulin, et le meunier procédait à sa vente comme pour les moutures. Les années où le blé était rare, il le vendait aux habitants, et le reste du temps aux boulangers ou aux marchands. Mais les années de disette, le blé de rentes se vendait en ville aux boulangers, à l'époque des semences, lorsque ceux-ci avaient de la peine à en trouver, et les années d'abondance, aux marchands, de manière que le Séminaire se trouve déchargé des frais de transport et de la fourniture des poches ${ }^{55}$. Les dîmes en

53 C. Objois. Les meuniers dans la seigneurie de l'île de Montréal au XVIIIe siècle (mémoire de maîtrise, Dpt d'histoire de l'Université de Montréal, 1981); et ASSSM, tiroir 45 , section 10 , dossier 1 .

54 C. Objois a inventorié 74 baux de moulins à farine entre 1685 et 1800, dont 44 moyennant une quantité de mouture fixe, et les autres, un pourcentage variable du produit du moulin. Op. cit., 238 et 71-72.

55 ASSSM, tiroir 91, no 40, vol. 3, Bleds de dixmes et de rentes, fol. $8, \mathrm{r}^{\circ}$ : point 3 et fol. $8 \mathrm{v}^{\circ}$ : point 9; ibidem, Bled et hangard à St Sulpice, fol. $8 \mathrm{v}^{\circ} 2$ : point 5 . 
nature, par contre, étaient montées par chaque habitant directement dans le grenier du Séminaire en ville ${ }^{56}$.

Les problèmes de transport au Canada, à courte comme à longue distance, n'ont cessé de faire l'objet d'ordonnances nombreuses sous les deux régimes, pour améliorer aussi bien l'état des chemins en été et en hiver, que les véhicules eux-mêmes. Ainsi, le Séminaire devait-il défrayer les meuniers qui lui amenaient son grain de consommation en ville, réduire de 10 sols le prix de vente du minot de blé de sa seigneurie du lac des Deux-Montagnes, et de 5 sols celui de sa seigneurie de Saint-Sulpice, lorsque les clients allaient chercher eux-mêmes le blé qu'ils y achetaient.

Entre 1796 et 1837 , le Séminaire a commercialisé annuellement de 2527 à 8489 minots de blé de mouture (environ 986 à $3310 \mathrm{hl}$, sur la base de 1 minot = 391 ), et de 922 à 4241 minots de blé de rentes et de dîmes (env. 360 à 1654 hl). D'une manière générale, l'essentiel de la récolte était en partie consommé et en partie vendu dans l'année, à cause des conditions de conservation rendues difficiles par les pointes de chaleurs et d'humidité de l'été. En hiver, le gel favorisait la conservation du grain, mais réduisait son volume, et, partant, le bénéfice à la vente. D'après l'économe chargé de la surveillance du grenier du Séminaire au «chemin couvert», qui était une galerie fermée reliant le Séminaire à l'église par-dessus l'entrée du cimetière ${ }^{57}$ :

comme ce bled est fort exposé aux rats et à la sécheresse, il faut le faire enlever le plus tôt qu'on peut. Si, par des raisons, on ne vend pas le bled de bonne heure, il faut avoir soin de le faire remuer tous les mois, de crainte qu'il ne chauffe.

Il arrivait à l'économe de signaler dans les comptes une augmentation du volume du blé conservé ${ }^{58}$.

Nous sommes cependant surpris de voir, à la fin de l'exercice annuel, en général à la Saint-Michel (29 septembre), les quantités de blé resté invendu dans les moulins, même en période de manque et de cherté. En 1801, par exemple, le prix maximum du minot de blé atteignait les 12 livres, et il restait encore à la fin de l'exercice

56 Cf. règlement du 4 septembre 1667 , où il fut arrêté que «les dîmes ne s'y payeraient à l'avenir que des grains seulement à raison du $26 \mathrm{e}$ minot, en considération de ce que les habitans seraient tenus de l'engranger, battre, vanner et porter au presbytère», Edits, ordonnances royaux..., op. cit., II: 133. 4 et 5 .

57 ASSSM, tiroir 91 , no 40 , vol. 3, Bled de dixmes et de rentes, fol. $8 \mathrm{r}^{\circ}$ : points

58 «dans les hangards, le bled non seulement ne diminue pas de volume, mais il renfle, et toujours il y a un Bon (excédent) quand on le mesure pour l'embarquer. Il faut avoir soin de se faire tenir compte de ce bon», ibidem, Bled et hangard à St Sulpice, fol. $8 \mathrm{v}^{\circ} 2$ : point 3; cf, également supra l'ordonnance de 1750 rendue contre la fraude des maîtres de barques. 
plus du sixième du produit de l'année; en 1813, alors que le minot était à 19 livres et en 1816 à 21 livres, il restait dans les deux cas encore un neuvième des moutures reçues durant l'année. En 1825, bien que les prix fussent bas depuis quelques années, aux environs de 6 livres le minot, la fin de l'exercice se solda par un invendu dans les moulins de plus du tiers de leur produit en blé. La mévente des blés de rentes et de dîmes, comptabilisée à part, était par contre minime. Cela tenait peut-être au fait qu'on séparait le «beau bled» de dîmes du mauvais, qui était lavé ou donné aux volailles, et que le portier avait ordre de refuser le mauvais blé de rentes, «tout bled taché, pointé, mal vanné ${ }^{59}$. Ces blés, pour ainsi dire sélectionnés, pouvaient constituer un lot d'une qualité plus recherchée.

Maîtres d'une partie des grains des censitaires de trois seigneuries, les Sulpiciens tenaient entre leurs mains un levier important de l'approvisionnement en blé de Montréal. Les livres de comptes avec leurs meuniers signalent, à côté des ventes aux boulangers, aux marchands et aux particuliers, la part de blé que le Séminaire réservait à sa propre consommation, à celle du collège du petit Séminaire et à celle des fermiers de ses deux domaines de la Montagne et de Saint-Gabriel, plus tard également à celui de la maison sous les noyers. Il en fournissait aussi, à titre gracieux, pour le pain des pauvres de l'Hôtel-Dieu et de l'Hôpital général. Si nous évaluons, aux dates extrêmes pour lesquelles nous avons déjà mesuré l'importance des moutures invendues dans les moulins, la part des grains donnés ou consommés par le Séminaire, celle-ci représenterait en 1801, année de prix fort, un peu plus du quart du produit annuel des moulins, et n'en serait plus que le huitième à peu près en 1825 , année de bas prix. Cette comparaison nous montre que le Séminaire satisfaisait à ses engagements avant de rechercher son profit et qu'il assumait l'approvisionnement en blé d'une partie au moins de la population indigente de Montréal. Le procureur notait encore souvent dans les dépenses de son Journal, «donné à un pauvre, en bled...». Les mauvaises années, toutefois, malgré la réduction considérable des quantités de blé commercialisable, le Séminaire réalisait quand même son meilleur bénéfice grâce à la conjoncture et aux prix élevés du grain.

\section{La boulangerie à Montréal}

Les débuts de la boulangerie à Montréal ont été modestes, à la mesure des besoins et des moyens de la population. En 1687, la première ordonnance concernant l'industrie du pain prévoyait, en effet, que:

59 ASSSM, tiroir 91, no 40, vol. 3, Bled de dixmes et de rentes, fol. $8 \mathrm{r}^{\circ}$ : points 
chacun conservera la liberté de cuire du pain, mais pour son usage seulement... (et) afin que le public (parmi lequel il fallait compter les aubergistes et les cabaretiers qui donnaient du pain à leurs hôtes) soit servi par des gens réputés honnêtes, les boulangers devront obtenir un permis de vente de M. le Juge et leur nombre sera limité. ${ }^{60}$

À en croire le renouvellement de cette dernière disposition quelques années plus tard, elle devait également être destinée à «lutter contre certaines personnes qui s'ingéraient de vendre du pain et qui parcouraient même les côtes autour de Montréal pour acheter le blé à un prix plus élevé qu'il ne valait» ${ }^{61}$. Les difficultés à s'approvisionner en blé entraînaient deux sortes de handicaps pour la boulangerie: l'abandon du commerce, lorsque le blé venait à manquer, comme ce fut le cas le 22 janvier 1690 à Québec, où deux boulangers durent renoncer à leur «société de boulangerie» faute de blé; la réduction du bénéfice, voire le travail à perte, si le blé se vendait dans la pratique au-dessus du prix fixé, ce qui avait amené les boulangers de Montréal à se plaindre auprès du bailli en septembre $1688^{62}$.

En 1710, il n'étaient encore que quatre à exercer officiellement à Montréal, pour une population estimée trois ans plus tôt à 1327 habitants dans la ville et les faubourgs ${ }^{63}$. Mais la même année, l'intendant adjoignait à ces boulangers un cinquième, puis quatre autres encore, car «la présence d'un grand nombre de sauvages dans Montréal rendait cette mesure nécessaire» ${ }^{64}$. En 1743, vu la rareté du blé, leur nombre fut réduit à sept, seuls autorisés à vendre du pain à Montréal; de ce fait, «le métier de boulanger était redevenu un métier fermé» ${ }^{65}$. Un peu plus d'un demi-siècle plus tard, Thomas Doige signalait 46 boulangers dans son Annuaire des commerçants résidant à Montréal, et 50 l'année suivante. Le premier recensement de population en 1825 indiquait 98 boulangers pour 22540 personnes dans la ville et les faubourgs, et identifiait 38 d'entre eux ${ }^{66}$. Le rapport entre le nombre de boulangers et celui de la population, entre les années $1707 / 10$ et 1825 , indique une réduction de plus de 100 personnes par boulanger (de 338 à 230).

80 .

60 E.-Z. Massicotte, «La boulangerie à Montréal avant 1760», BRH. 47, 3 (1941):

616 août 1695, J.-N. Fauteux, op. cit., II: 370.

62 Ibidem: 369.

63 L. Dechêne, «La croissance de Montréal...", op. cit., 164.

64 J.-N. Fauteux, op cit. II: 374, citation d'après Faillon.

65 Ibidem: 375.

66 C. Perrault, Montréal en 1825 (Montréal, 1977); et GRSM, op. cit.; les noms de ces 38 boulangers nous ont été aimablement communiqués par J.-Cl. Robert, grâce à l'informatisation des listes nominatives du recensement. 
Cette tendance, qui ne vaut que par le sens qu'elle indique et auquel on pouvait d'ailleurs s'attendre, soulève en fait la question du four à pain familial. L'existence d'un four à pain intérieur, qui est construit "dans une paroi de la cheminée» ${ }^{67}$, est attestée en milieu urbain par des contrats notariés portant sur la réparation ou la construction de ces fours par des ouvriers rémunérés ${ }^{68}$.

Le deuxième recensement de 1831 permet de relever le nom de 83 boulangers seulement pour la ville et les faubourgs, alors que la ville comptait 27248 habitants. Cette réduction du nombre de boulangers correspondant à un accroissement de population donne à penser que tous les boulangers en fonction n'ont pas été recensés. En effet, le recensement de 1831 ne reprend que les renseignements qui concernent la profession du chef de famille ${ }^{69}$.

L'étude des quelque 250 acheteurs du blé de mouture, de rentes et de dîmes du Séminaire de Saint-Sulpice, nommés dans les comptes des moulins banaux et dans le Journal du procureur, est en cours. Une première analyse, portant uniquement sur les boulangers désignés dans les comptes en tant que tels ${ }^{70}$, en a signalé 27 pour la période 1800-39; sept d'entre eux ont été retrouvés dans les actes notariés aux ANQM, quatorze dans les Annuaires de Doige, huit dans le recensement de 1825 et six dans celui de 1831. Parmi les 13 inventaires après décès étudiés aux archives à ce jour et dont 6 concernent des clients du Séminaire, les inventaires qui ont été établis au décès des deux épouses de Joseph Charlebois, maître boulanger au faubourg St-Joseph et client du Séminaire entre 1799 et 1813 , témoignent de la réussite et de l'importance prise par ce boulanger qui est toujours actif en 1813. Rappelons que les inventaires dressés du vivant du boulanger sont plus révélateurs sur l'activité boulangère que lorsqu'ils interviennent après sa mort. En 1805, au décès de la femme Marie Archange Gaudry, le couple n'était pas propriétaire de la maison où il habitait et il n'y avait pas de boulangerie, mais "une salle en entrant» qui en tenait lieu; en 1810, à la mort de Marie Louise Marie dite Ste Marie, le couple avait fait construire «une maison en pierre... avec boulangerie et four dessus», ainsi qu' «une voûte en pierre à deux étages» qui ser-

67 R.-L. Seguin, Les ustensiles en Nouvelle-France (Ottawa, 1972), 5 note 6.

68 L. Boily et J.-F. Blanchette, Les fours à pain au Québec (Ottawa, 1976), 4.

69 Nous avons cherché à enrichir ces informations par un premier sondage dans douze fonds notariaux aux Archives Nationales du Québec à Montréal (ANQM) qui, par le truchement d'actes divers comme des inventaires après décès, des actes de mariage, des contrats d'apprentissage, de vente, des baux, etc., nous ont fourni les noms de 27 boulangers pour Montréal, ville et faubourgs, entre 1772 et 1834 . Le relevé des inventaires après décès nous a été aimablement communiqué par J.-P. Wallot et son assistante de recherche.

70 Par exemple, les Comptes des Négociants, Boulangers et autres qui achètent le bled et autres grains, ASSSM, no 195, VIe partie du Livre de compte tenu par le Procureur du Séminaire de Montréal. 
vait d'entrepôt ${ }^{71}$. L'estimation totale des biens meubles et immeubles avait plus que doublé entre les deux dates.

La clôture d'inventaire des biens en communauté avec sa défunte première femme et continuée avec la seconde par le maitre boulanger Julien Perrau/1/d $/ \mathrm{t}$ du faubourg Saint-Laurent, client du séminaire de 1800 à 1812, signale, au chapitre des dettes actives, le nom des clients qui leur ont acheté du pain à crédit; les dettes pour pain les plus élevées atteignent 60 livres $^{72}$. Voici, à titre indicatif, le prix du pain arrêté à la Session spéciale de la Paix du ler juin de la même année ${ }^{73}$ : «It is ordered that the price of bread for the present month be as follow. The white loaf of 4 (pounds) to be One Shilling. The Brown Dto of 6 (pounds) to be thirtheen pence.» En reprenant la consommation de 2 livres de pain par jour, indiquée par l'intendant en 1706, on obtiendrait un arriéré approximatif de $4 \frac{1}{2}$ mois de pain bis pour chacun des quatre clients les plus endettés auprès de Julien Perrault, ce qui ne représenterait plus qu' $1 \frac{1 / 2}{2}$ mois d'arriéré si l'on comptait un pain bis de 6 livres par jour pour une famille de 4 ou 5 personnes par exemple.

Joseph Berlinguet, maître boulanger au faubourg SaintLaurent et client du Séminaire en 1799, procéda en 1802 à des ventes de grain importantes, allant de 1500 à 3000 minots, à des négociants de Montréal ${ }^{74}$. En effet, en plus des deux qualités de pain, blanc ou de fine fleur, et bis-blanc (au XVIIe siècle, on mangeait encore normalement du pain brun), et des biscuits et des galettes qu'ils faisaient ordinairement, les boulangers semblent également avoir vendu en temps normal du blé et de la farine ${ }^{75}$. Comme les meuniers, les boulangers pouvaient constituer un relais dans l'approvisionnement en blé de Montréal. En juillet 1804, Joseph Berlinguet engageait pour trois ans un apprenti de 18 ans de la rivière Chambly, mais un an et demi plus tard celui-ci voyait annuler son contrat «sans aucun dédommagement de part et d'autre» malgré les conditions arrêtées précédemment par les deux parties, parce que son patron avait été «interdit le 23 décembre courant (année 1805) pour cause de dérangement d'esprit» ${ }^{76}$. Le compte de curatelle, rendu le 28 mars suivant par le curateur de Berlinguet au tuteur des enfants mineurs qu'il avait eus de son pre-

71 ANQM, J. Papineau, no 3648 du 16 janvier 1805, et no 4140 du ler août 1810 .

72 Ibidem, J.-M. Cadieux, no 195 du 9 juin 1812.

73 Archives de la ville de Montréal, Procès-verbaux des Sessions spéciales de la paix, 1796-1839, II: 249.

74 ANQM, Louis Chaboillez, no 5115 du 24 février 1802, no 5222 du 23 avril 1802 et no 5849 du 20 avril 1803 .

${ }_{75}$ Cf. l'interdiction qui leur fut faite en 1714, «vu la récolte peu abondante... de faire des biscuits, même de vendre du blé et de la farine», E.-Z. Massicotte, op. cit., 82.

76 ANQM. Ls Chaboillez, no 6556 du 23 juillet 1804, et J.-M. Mondelet, no 2984 du 28 décembre 1805 . 
mier mariage, faisait état des travaux réalisés à un emplacement au faubourg Saint-Laurent pour la «couverture de deux fours, 42 livres... (et l'aménagement d') 1 four de briques contenant environ 80 pains, la masse, clôture et entourage, et 1 cheminée, 1200 livres» ${ }^{77}$. C'est la première indication que nous rencontrons sur la capacité d'un four et sur la présence de plusieurs fours chez un même boulanger. Au chapitre 2ème des recettes de ce compte figure encore l'indication à ce même emplacement $d$ ' $« 1$ moulin à cheval pour farine». Cette mention remettrait-elle en question la déclaration de l'économe du Séminaire sur la seconde mouture que les boulangers faisaient gagner aux seigneurs de Montréal, en portant moudre à leurs moulins banaux le blé de mouture ou de rentes qu'ils y avaient déjà acheté?

Aux informations que les actes notariés sont susceptibles de livrer sur le rôle et les moyens dont disposaient les boulangers pour approvisionner Montréal en blé, en farine et en pain, viennent s'ajouter les précisions fournies par d'autres sources. Les Annuaires de Doige donnaient, avec les nom et prénom des intéressés, leur adresse en ville ou dans les faubourgs, avec la rue, voire le numéro (ce qui serait de pure fantaisie). Le recensement de 1825 indiquait le quartier ou le faubourg mais non la rue; il comportait également l'âge du boulanger, la composition de sa famille, les personnes étrangères, les employés et parfois les conditions d'habitation. Un peu plus sommaire, le recensement de 1831 ajoutait cependant à cette liste la présence des bêtes à cornes et surtout celle des chevaux, détail important, non seulement pour mesurer l'indépendance du boulanger dans son approvisionnement en blé et en farine, mais éventuellement aussi pour savoir s'il pouvait faire partie de ceux qui assuraient la distribution du pain à domicile dans les quartiers dépourvus de boulangerie. Tant que Montréal ne fut pas étendu, «les boulangers ne furent que tenus d'avoir en leur établissement le nombre de pains qu'il fallait pour leur clientèle ordinaire, mais avec l'année 1743, on obligea les boulangers qui probablement desservaient la ville et les faubourgs de plus en plus populeux, à s'arranger pour que le pain fût distribué par l'un d'eux, le matin à huit heures, et l'après-midi à trois heures ${ }^{78}$. D'après l'estimation de L. Dechêne, la ville et les faubourgs auraient compté 3575 habitants en $1741^{79}$. En 1825, alors que la population comptait 6,3 fois plus d'habitants, pas loin des deux tiers des boulangers connus étaient concentrés au faubourg Saint-Laurent; en 1831, avec un taux d'accroissement de la population de 3,2\%, l'essentiel

77 Idem, no 3093 du 28 mars 1806.

78 E.-Z. Massicotte, op. cit., 82.

79 L. Dechêne, Habitants et marchands..., op. cit., 164, Tableau 1. 
des boulangers identifiés restait massé dans le même faubourg, tandis que le quartier centre-ville et le faubourg Saint-Jacques apparemment n'en auraient pas compté. Dans ces conditions, la nécessité de distribuer le pain à domicile devenait impérieuse. Après la Conquête, l'ordonnance de 1764, qui visait à «prévenir les fraudes dans le poids et l'Assise du Pain», autorisait les «Gréffiers (des marchés)... à arrêter et examiner le Pain qui sera porté par les rües, que ce soit pour une vente immédiate, ou pour en fournir à leurs coutumes» ${ }^{80}$. Nous n'avons pas identifié dans les documents la tenue de marchés au pain comme à Paris, par exemple. Le texte de cette ordonnance évoquerait plutôt, à côté de la livraison à domicile, une vente ambulante dans les rues de Montréal. Pour autant que la localisation plus rigoureuse des boulangeries soit possible, leur regroupement par quartier esquissé dans les sources laisse déjà entrevoir de fortes inégalités d'achalandage suivant les quartiers de la ville et les faubourgs. Si cela devait être le cas, la situation à Montréal dénoterait la liberté laissée aux boulangers dans le choix des moyens d'achalander la population, alors qu'à Bologne, par exemple, le système municipal, dont les contraintes pesaient sur la corporation des boulangers, en hiérarchisait pour ainsi dire les activités ${ }^{81}$.

En l'absence d'une mercuriale des prix et d'un enregistrement des transactions de marché, il nous a paru indispensable d'étudier les fonctions du blé dans une région devenue productrice, avant de suivre les filières d'approvisionnement de Montréal.

La culture du froment a été privilégiée par le système agricole mis en place. Mais après avoir assuré le principe de l'autonomie alimentaire de la colonie, elle n'a pas bénéficié des conditions nécessaires à son insertion dans le réseau d'échanges internationaux auquel certains pays européens participaient depuis le Moyen âge. L'intérêt et les moyens des petits exploitants n'ont pas été suffisamment ni durablement sollicités par les débouchés du marché, de sorte que la monoculture du blé a développé différentes fonctions, étroitement interdépendantes, à une époque où l'Europe privilégiait celle du commerce. Comme par une ironie de l'histoire - ou ne serait-ce pas plutôt en fonction de l'évolution historique d'un ensemble de rapports de force? - Montréal allait occuper la première place dans l'industrie et le commerce des farines au I: 14 et 15 .

803 septembre 1764, Ordonnances, faites pour la Province de Québec..., op. cit.,

${ }_{81}$ A. Guenzi, «Un mercato regolato: pane e fornai a Bologna in età moderna», Quaderni storici, 37 (1978): 370-397. 
Canada, au moment du déclin de la céréaliculture au Québec. Ce renversement de situation engagea des auteurs de la fin du XIXe siècle, qui vraisemblablement avaient vécu la transformation industrielle de Montréal, à évoquer cette nouvelle réalité avec ses perspectives d'avenir ${ }^{82}$ :

La ville contient les plus grands moulins de tout le Canada... La farine de Montréal est expédiée dans toutes les parties du Canada de Vancouver à Halifax, de même en Grande Bretagne, à Anvers et Hambourg, et le chemin de fer du Pacifique a ouvert un vaste débouché avec la Chine et le Japon...

Mais, poursuivaient-ils, «antérieurement il y a quinze ans, l'industrie des moulins à farine avait fait bien peu d'avance».

En effet, dès les années 1837, lorsque le Séminaire commença à se défaire de ses moulins, la masse de blé qui y était traitée avait fondu par rapport à ce qu'elle avait été au début des comptes avec les meuniers que nous avons vus.

Au cours de cette étude, qui porte sur la période seigneuriale à Montréal, il nous est apparu que le système d'approvisionnement de la ville était peu contraignant, comparé à celui de la police française des grains ou aux responsabilités municipales dans les villes du Saint-Empire. L'analyse de ce système met en évidence la dimension politique du problème, dans la mesure où le plein exercice du droit de banalité permettait aux seigneurs de Montréal de substituer en quelque sorte plusieurs points de vente périphériques à une halle aux grains centrale. Ces points de vente étaient constitués par les moulins banaux, où le prélèvement du blé de mouture renouvelait sans cesse les stocks. La position-clé du Séminaire de Saint-Sulpice à Montréal ne doit cependant pas faire perdre de vue les initiatives des particuliers, ni, surtout, les apports des seigneuries voisines. Le développement de la boulangerie, enfin, assura en partie, avec les meuniers, les relais d'approvisionnement en grain, en farine et en pain de la population urbaine, au cours de ses différentes phases de croissance. merce, industrie. Depuis 1535 à 1889 (Montréal, 1890), 97. 